\title{
ESTIMATIVA DA PRODUÇÃO DE FORRAGEM EM PASTAGEM DE BRACHIARIA DECUMBENS
}

\author{
FORAGE PRODUCTION ESTIMATE IN BRACHIARIA DECUMBENS PASTURE \\ Costa, B.M. da ${ }^{1 \mathrm{~A}}$, C.A.S. Ledo ${ }^{2}$, M.C. Silva ${ }^{1 \mathrm{~B}}$ e V.I. Teixeira ${ }^{1 \mathrm{C}}$
}

${ }^{1}$ Centro de Ciências Agrárias, Ambientais e Biológicas. UFRB. Cep 44380-000 Cruz das Almas. Bahia. Brasil. Abeneditomc@hotmail.com; Bmaxagrufba@yahoo.com.br; 'vicente_teixeira@bol.com.br ${ }^{2}$ Embrapa, Mandioca e Fruticultura Tropical. CEP 44380-000 Cruz das Almas. Bahia. Brasil. ledo@cnpmf.embrapa.br

\section{PaLAVRas ChaVE ADICIONAIS}

Avaliação de pastagens. Dupla amostragem. Matéria verde.

\section{RESUMO}

Objetivou-se comparar os métodos visual, da altura do relvado e do corte na estimativa da produção de forragem em pastagem de Brachiaria decumbens Stapf., em área do Centro de Ciências Agrárias, Biológicas e Ambientais da UFRB. Nas estimativas de peso visual foram tomadas, dentro de uma moldura de ferro de $0,25 \mathrm{~m}^{2}, 10$ duplas amostras de peso visual $(x)$ e real $(y)$, sendo amostra 1= menor peso ou ausência de forragem; amostra 10= maior peso da forragem; amostras 2 a $9=$ pesos intermediários. Com os valores de estimativas visual $(x)$ e real $(y)$ realizou-se uma análise de regressão, observando-se uma relação linear entre as variáveis $x$ e y pelo teste $t(p<0,01)$, obtendo-se a equação $y=1,85+0,82 x$. Nas estimativas de forragem pelo método da altura do relvado tomou-se 10 duplas amostras, sendo 10 da altura média do relvado (x) e 10 do peso real (y) da forragem, tomadas dentro da citada moldura de ferro, obtendo-se a equação $y=1,55+0,41 x$ e verificando-se uma relação linear entre as duas variáveis $(p<0,01)$ pelo teste t. Em seguida, utilizando-se quatro transeções lineares paralelas, espaçadas de $15 \mathrm{~m}$, com a moldura de $0,25 \mathrm{~m}^{2}$ foram amostrados 25 pontos, distanciados de $5 \mathrm{~m}$, resultando em 100 pontos de amostragem nos três métodos avaliados. As equações de regressão encontradas foram usadas para fazer o ajustamento das estimativas de peso real (y) em relação às de peso visual $(x)$ e das medidas de altura média do relvado $(\mathrm{x})$. A análise de variância

Recibido: 23-4-07. Aceptado: 17-5-07.

\section{AdDitional KeYWORDS}

Pasture evaluation. Double sampling. Fresh matter.

não revelou significância pelo teste $F(p>0,05)$ entre os métodos avaliados. É possível estimar a produção de forragem de uma pastagem de capimbraquiária através de estimativas visuais e medidas de altura do relvado, utilizando uma equação de regressão linear.

\section{SUMMARY}

It was compared the visual estimate, foliage heights and cut methods in forage yield estimation of Brachiaria decumbens Stapf. pasture, in an area of the Agricultural, Environmental and Biological Sciences Center of UFRB. In the visual estimate method, forage estimations were done within an iron quadrat of $0.25 \mathrm{~m}^{2}, 10$ double samples of visual $(x)$ and true weight $(y)$, being sample 1= smaller forage weight or lack of forage; sample $10=$ higher forage weight; samples 2 to $9=$ intermediary weights. It was carried out a regression analysis with the values of visual $(x)$ and true $(\mathrm{y})$ estimates obtaining the equation $\mathrm{y}=$ $1.85+0.82 x$, and observing a linear relationship between the variables $x$ and $y$ using $t$ test. In the forage estimations using foliage height measurements it was done 10 double samples, being 10 of the mean foliage height $(x)$ and 10 of the true forage weight $(y)$, done within cited iron quadrat, obtaining the equation $y=1.55+0.41 x$ and verifying a linear relationship between the two variables $(p<0.01)$ by $t$ test. Afterwards, utilizing four

Arch. Zootec. 58 (221): 141-144. 2009. 
parallels linear transections, spaced of $15 \mathrm{~m}$, using the $0.25 \mathrm{~m}^{2}$ iron quadrat were sampled 25 points, spaced of $5 \mathrm{~m}$, resulting in 100 points of sampling comparing the three methods. There was not significance by F test ( $p>0.05$ ) among the evaluated methods, making possible to estimate forage yield of a $B$. decumbens pasture by visual estimates and foliage heights measurements, utilizing a linear regression equation.

\section{INTRODUÇÃO}

No manejo das pastagens é de primordial importância o conhecimento da produção de forragem atual, em $\mathrm{kg} / \mathrm{ha}$, para que se possa calcular a sua capacidade de suporte. O método mais utilizado na avaliação de pastagens consiste no corte e pesagem da biomassa vegetal. Este método é o mais preciso, porém é lento e trabalhoso, requerendo muito tempo e um grande número de amostras para se obter estimativas confiáveis. Felizmente, os pesquisadores estudaram métodos não destrutivos que envolvem duplas amostragens de estimativas visuais em relação ao peso da forragem (Cóser et al., 1980; Nascimento Jr. et al., 1982; Lopes et al., 2000) e de medidas de altura e peso do relvado (Bandinelli et al., 2003; Matos et al., 2004), possibilitando um grande número de observações e rapidez nas estimativas da produção de forragem da pastagem.

O objetivo deste estudo foi avaliar os métodos de estimativa visual, altura do relvado e do corte na estimativa da produção de forragem de uma pastagem de capimbraquiária (Brachiaria decumbens Stapf. cv. IPEAN), localizada em área de Tabuleiro, no Centro de Ciências Agrárias, Ambientais e Biológicas da UFRB, em Cruz das Almas, BA, Brasil.

\section{MATERIALE MÉTODOS}

As estimativas da produção de forragem da pastagem foram realizadas em área da Fazenda de Pecuária do Centro de Ciências Agrárias, Ambientais e Biológicas da
Universidade Federal do Recôncavo da Bahia, UFRB, no município de Cruz das Almas, BA, comparando-se os métodos: visual, altura do relvado e do corte. No método de estimativa visual foram tomadas 10 duplas amostras padrões, sendo 10 de estimativa visual (x) e 10 de peso real (y) da forragem. As 10 duplas amostras padrões de estimativa de peso visual (x) e real (y) foram obtidas do seguinte modo: amostra $1=$ menor peso ou ausência de forragem; amostra $10=$ maior peso da forragem; amostras 2 a $9=$ pesos intermediários entre 1 e 10.

Para utilização do método de estimativa visual o observador submeteu-se a um treinamento prévio no campo fazendo estimativas visuais de peso, seguidas do corte e pesagem da amostra para avaliar o grau de acerto da estimativa visual realizada. Após esse treinamento, foram tomadas 10 duplas amostras padrões, que foram analisadas estatisticamente por regressão para verificar se havia uma relação linear entre as variáveis x (peso visual) e y (peso real). No caso de haver significância entre essas duas variáveis $(\mathrm{p}<0,05)$, utilizou-se a equação de regressão linear do tipo $\mathrm{y}=\mathrm{a}+$ bx para ajustar as estimativas de peso real (y) através das estimativas de peso visual (x) ao longo das transeções lineares.

No método da altura do relvado foram também tomadas 10 duplas amostras padrões, sendo 10 de medidas da altura do relvado (x) e 10 de peso real (y) da forragem. As 10 duplas amostras padrões foram obtidas do seguinte modo: amostra $1=$ altura do relvado e peso da forragem zero, localizada em área com ausência total de forragem; amostra $10=$ maior altura do relvado e maior peso da forragem; amostras 2 a $9=$ alturas e pesos intermediários entre 1 e 10. As 10 duplas amostras padrões foram analisadas estatisticamente por regressão para se verificar se havia uma relação linear entre as variáveis x (altura do relvado) e y (peso real da forragem). Utilizou-se a equação de regressão do tipo linear $\mathrm{y}=\mathrm{a}+\mathrm{bx}$ para

Archivos de zootecnia vol. 58, núm. 221, p. 142. 
ajustar as estimativas de peso real (y) através das medidas de altura do relvado (x).

Nas estimativas de produção de forragem, nos três métodos avaliados, foram estabelecidas quatro transeções lineares paralelas, utilizando cordas de nylon, espaçadas de 10-15 m onde foram marcados 25 pontos, distantes $5 \mathrm{~m}$ um do outro, resultando em 100 pontos de amostragem. Para isso, em cada ponto de amostragem ao longo da corda registrou-se a altura média, a estimativa visual e o peso da forragem verde, utilizando uma moldura de ferro de $0,25 \mathrm{~m}^{2}$. $\mathrm{Na}$ análise dos resultados utilizou-se o programa Statistical Analysis System (SAS, 2000).

\section{RESULTADOSEDISCUSSÃO}

A análise de regressão demonstrou que há uma relação linear altamente significativa $(p<0,01)$ pelo teste $t$ entre as estimativas de matéria vede visual (x) e matéria verde real (y). Essa relação linear positiva entre esses parâmetros foi representada pela equação $y=1,85+0,82 x$ (figura 1$)$. O coeficiente de regressão $(b=0,82)$ significa que para um aumento de uma tonelada de matéria verde estimada visualmente há um correspondente aumento de $820 \mathrm{~kg}$ na matéria verde real. O coeficiente de correlação ( $\mathrm{r}=$ $0,91)$ para essas variáveis foi altamente significativo $(\mathrm{p}<0,01)$, concordando com o obtido por Cóser et al. (1980) que encontraram um valor de 0,95 em pastagem de milheto e sorgo. O coeficiente de determinação $\left(\mathrm{R}^{2}=0,83\right)$ mostra que as estimativas visuais de produção de matéria verde explicam $83 \%$ da variação existente nas estimativas reais de produção de matéria verde. Esse valor foi inferior aos coeficientes de determinação $\left(\mathrm{R}^{2}\right)$ de 0,91 a 0,98 , obtidos por Nascimento Jr. et al. (1982) nas estimativas de matéria verde pelo método visual, porém foi superior ao $\mathrm{R}^{2}$ de 0,735 observado por Bandinelli et al. (2003).

A análise de regressão demonstrou que há uma relação linear altamente significati-

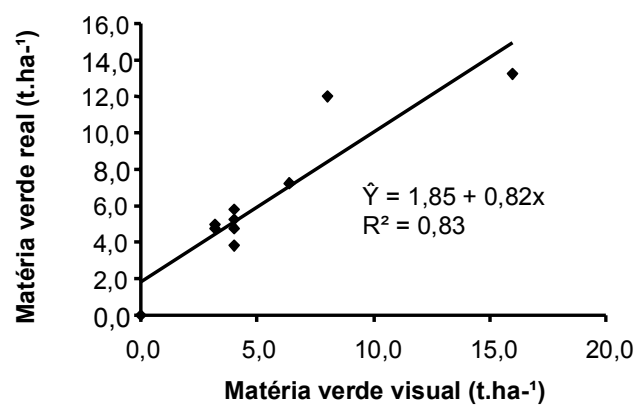

Figura 1. Regressão linear da matéria verde real (y) em função da estimativa da matéria verde visual $(x)$ em pastagem de Brachiaria decumbens Stapf. (Linear regression of actual green matter $(y)$ in function of the estimated visual green matter (x) in Brachiaria decumbens Stapf. pasture).

va $(\mathrm{p}<0,01)$ pelo teste $t$ entre as medidas de altura do relvado $(\mathrm{x})$ e de peso da matéria verde real (y). Essa relação linear positiva entre esses parâmetros foi representada pela equação $y=1,55+0,41 \times$ (figura 2). O coeficiente de regressão $(b=0,41)$ significa que para um aumento de uma tonelada de matéria verde estimada por medida da altura do relvado há um correspondente aumento de $410 \mathrm{~kg}$ na matéria verde real. O coeficiente de correlação $(\mathrm{r}=0,94)$ para essas variáveis foi altamente significativo $(p<0,01)$. O coefi-

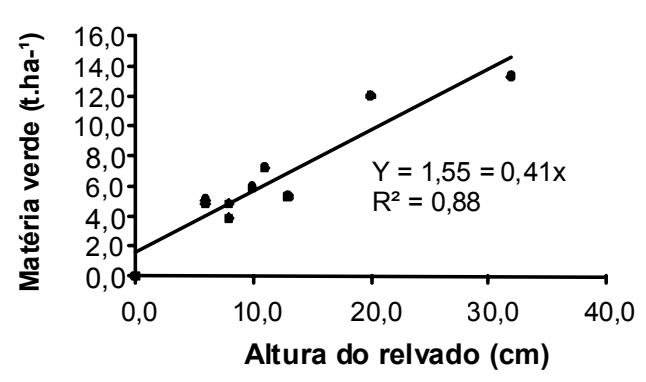

Figura 2. Regressão linear da matéria verde real (y) em função da altura do relvado (x) em pastagem de Brachiaria decumbens Stapf. (Linear regression of the actual green matter $(y)$ in function of the foliage height $(x)$ in Brachiaria decumbens Stapf. pasture). 


\section{COSTA, LEDO, SILVAE TEIXEIRA}

ciente de determinação $\left(\mathrm{R}^{2}=0,88\right)$ mostra que as estimativas de produção de matéria verde utilizando medidas de altura do relvado explicam $88 \%$ da variação existente nas estimativas de matéria verde real. Esse valor de $\left(\mathrm{R}^{2}\right)$ foi superior a 0,506 observado por Bandinelli et al. (2003), superior a 0,586 e 0,766 e inferior a 0,937 , obtidos por Matos et al. (2004), respectivamente, no primeiro, segundo e terceiro períodos de avaliação.

As 100 estimativas de matéria verde pelos métodos visual e da altura do relvado foram respectivamente corrigidas para obtenção da matéria verde real, utilizando as equações $\mathrm{y}=1,85+0,82 \mathrm{x}$ (método visual) e $y=1,55+0,41 x$ (método da altura). Esses

\section{BIBLIOGRAFIA}

Bandinelli, D.G., F.L.F. de Quadros, C.E.N. Martins, N.B. Trevisan, A.C.F. da Silva, A.R. Maixner, M.S. Brum e L.F.C. Simões. 2003. Comparação de métodos para estimativa da massa de forragem em gramíneas de estação fria. Em: Reunião Anual da Sociedade Brasileira de Zootecnia, 40, 2003, Santa Maria. Anais ... SBZ. Santa Maria.

Cóser, A.C., A.L. Collares e G.E. Marasching. 1980. Estimativa visual da forragem disponível como critério para ajuste da carga animal em pastagens. Rev. Soc. Bras. Zootecn., 9: 643655.

Lopes, R.S., D.M. da Fonseca, A.C. Cóser, D. Nascimento Jr., C.E. Martins e J.A. Obeid. 2000. Avaliação de métodos para estimação da disponibilidade de forragem em pastagem de valores quando comparados por análise de variância com as 100 estimativas de matéria verde, obtidas pelo método do corte, revelaram não haver significância estatística ( $p>0,05)$ entre os métodos avaliados.

\section{CONCLUSÕES}

É possível estimar a produção de forragem em pastagem de Brachiaria decumbens utilizando o método de estimativa visual ou por medidas de altura do relvado com dupla amostragem. Ambos o métodos necessitam de uma equação de regressão linear do tipo $\mathrm{y}=\mathrm{a}+\mathrm{bx}$ para ajustamento das estimativas.

capim-elefante. Rev. Soc. Bras. Zootecn., 29: 40-47.

Matos, R.S., P.A. Carvalho, R.L. Oliveira, M.R. Freitas, J.P. Velho e J. Viégas. 2004. Estimativa da produção de matéria seca de gramíneas anuais de inverno através da altura média da forragem. Em: Reunião Anual da Sociedade Brasileira de Zootecnia, 41, 2004, Campo Grande. Anais ... SBZ. Campo Grande.

Nascimento Jr., D., A. Ludwig e J.D. Moreira. 1982. Avaliação do método de dupla amostragem na estimativa da matéria verde disponível em pastagens naturais de Viçosa, MG. Rev. Soc. Bras. Zootecn., 11: 502-511.

SAS. 2000. Statistical Analysis System Institute Inc. SAS/STAT User's .Guide. V. 8.0, vol. I. SAS Institute, Inc. Cary NC. 